\title{
Hydrodynamic correlations of viscoelastic fluids by multiparticle collision dynamics simulations
}

Cite as: J. Chem. Phys. 151, 194110 (2019); https://doi.org/10.1063/1.5126082

Submitted: 29 August 2019. Accepted: 31 October 2019. Published Online: 20 November 2019

David Toneian (D), Gerhard Kahl (D), Gerhard Gompper (D), and Roland G. Winkler (D)

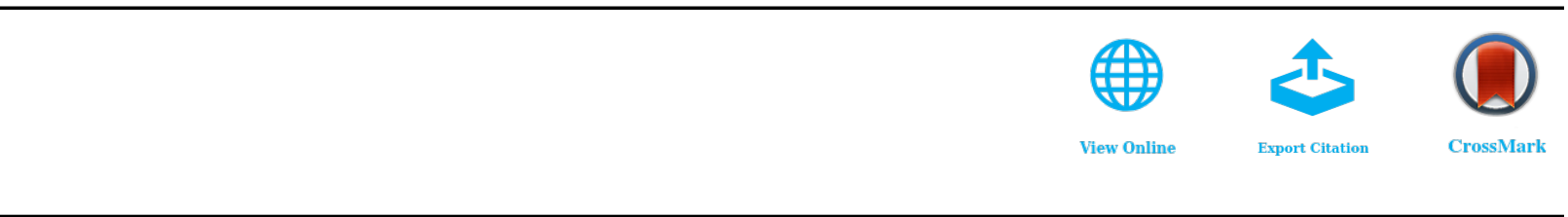




\title{
Hydrodynamic correlations of viscoelastic fluids by multiparticle collision dynamics simulations
}

\author{
Cite as: J. Chem. Phys. 151, 194110 (2019); doi: 10.1063/1.5126082 \\ Submitted: 29 August 2019 - Accepted: 31 October 2019 • \\ Published Online: 20 November 2019
}

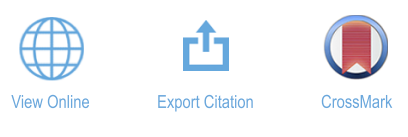

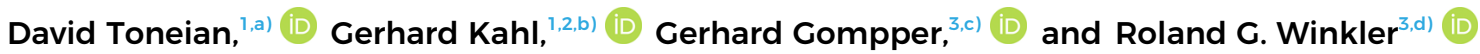

\begin{abstract}
AFFILIATIONS
${ }^{1}$ Institute for Theoretical Physics, TU Wien, Wiedner Hauptstraße 8-10, 1040 Wien, Austria

${ }^{2}$ Center for Computational Materials Science (CMS), TU Wien, Wiedner Hauptstraße 8-10, 1040 Wien, Austria

${ }^{3}$ Theoretical Soft Matter and Biophysics, Institute of Complex Systems and Institute for Advanced Simulation, Forschungszentrum Jülich, 52425 Jülich, Germany
\end{abstract}

a)Electronic mail: david@toneian.com

${ }^{b)}$ Electronic mail: gerhard.kahl@tuwien.ac.at

${ }^{c)}$ Electronic mail: g.gompper@fz-juelich.de

d) Electronic mail: r.winkler@fz-juelich.de

\begin{abstract}
The emergent fluctuating hydrodynamics of a viscoelastic fluid modeled by the multiparticle collision dynamics (MPC) approach is studied. The fluid is composed of flexible, Gaussian phantom polymers that interact by local momentum-conserving stochastic MPCs. For comparison, the analytical solution of the linearized Navier-Stokes equation is calculated, where viscoelasticity is taken into account by a time-dependent shear relaxation modulus. The fluid properties are characterized by the transverse velocity autocorrelation function in Fourier space as well as in real space. Various polymer lengths are considered-from dumbbells to (near-)continuous polymers. Viscoelasticity affects the fluid properties and leads to strong correlations, which overall decay exponentially in Fourier space. In real space, the center-of-mass velocity autocorrelation function of individual polymers exhibits a long-time tail, independent of the polymer length, which decays as $t^{-3 / 2}$, similar to a Newtonian fluid, in the asymptotic limit $t \rightarrow \infty$. Moreover, for long polymers, an additional power-law decay appears at time scales shorter than the longest polymer relaxation time with the same time dependence, but negative correlations, and the polymer length dependence $L^{-1 / 2}$. Good agreement is found between the analytical and simulation results.
\end{abstract}

Published under license by AIP Publishing. https://doi.org/10.1063/1.5126082

\section{INTRODUCTION}

Soft matter and complex fluids are composed of a broad range of nano- to microscale objects. Such systems are typically easily deformable, with characteristic energies on the order of the thermal energy and correspondingly long relaxation times, and entropic degrees of freedom play an important role. ${ }^{1-3}$ Paradigmatic examples of soft matter are biological cells (containing a wide range of polymeric and colloidal ingredients ${ }^{4,5}$ ), blood, solutions of polymers, emulsions, and suspensions of colloidal particles. ${ }^{6,7}$ The majority of these suspensions are viscoelastic rather than Newtonian, combining the viscous properties of fluids with the elastic characteristics of solids. ${ }^{6,8-11}$

Computer simulations are a valuable tool for gaining insight into the viscoelastic properties of complex fluids. ${ }^{12}$ Of particular interest are mesoscale simulation techniques, which account for hydrodynamic interactions and are able to bridge the length- and time-scale gap between fluid degrees of freedom and those of the embedded (polymeric) particles. ${ }^{13,14}$ Established mesoscale techniques are the lattice Boltzmann (LB) method, ${ }^{15-18}$ dissipative particle dynamics (DPD), ${ }^{19,20}$ and the multiparticle collision dynamics approach (MPC). ${ }^{21-23}$ Viscoelasticity is incorporated in different ways in the various simulation approaches. The LB method describes a fluid in terms of a spatially discretized probability density, whose dynamics progresses via the Boltzmann equation. ${ }^{17,18}$ Viscoelasticity is incorporated by extending the stress tensor by a viscous-stress contribution, e.g., the Maxwell model, ${ }^{6,24,25}$ and taking this stress into account as a body force in the discretized propagation equation. ${ }^{24,25}$ In contrast, DPD and MPC are particle-based simulation approaches, where the bare fluid is represented by point particles 
and a complex fluid by additional suspended objects such as colloids, polymers, membranes, or cells. In the latter approaches, viscoelasticity emerges as a consequence of the interactions between the embedded objects. Examples of viscoelastic DPD simulations are studies of blood cells ${ }^{26}$ and star polymers ${ }^{27}$ in flow. For MPC, the rheological properties of linear, branched, and star polymers $^{27-33}$ have been investigated, as well as those of cells and vesicles. $^{34}$

Alternatively, viscoelastic fluids can be modeled by an ensemble of more complex entities, directly representing a viscoelastic fluid rather than a viscoelastic suspension. DPD and MPC viscoelastic fluids can be modeled by linearly connected DPD and MPC particles, respectively. The simplest viscoelastic unit is a dumbbell. The extension of the original DPD approach to a dumbbell fluid is presented in Ref. 35 and to even longer polymers in Ref. 36. Similarly, the properties of MPC dumbbell fluids of different complexities are studied in Refs. 13, 14, 37, and 38.

This representation of a viscoelastic fluid via an ensemble of linear elastic polymers raises a number of fundamental questions on hydrodynamic interactions in such systems. Traditionally, it is assumed that hydrodynamic interactions are screened in polymer melts and that the properties of individual polymers in the melt are well described by the Rouse model. ${ }^{10,39}$ Screening is assumed to emerge by the excluded-volume interactions between the polymers. Conversely, analytical considerations show that in melts of phantom polymers, i.e., polymers without excluded-volume interactions, hydrodynamic interactions are unscreened.

Recent computer simulations and theoretical studies of unentangled polymer melts including excluded-volume interactions raise considerable doubts on these simple pictures, since the studies show clear evidence of a long-time tail in the polymer velocity correlation function, indicative of unscreened hydrodynamic interactions. $^{41,42}$

In this article, we study the properties of a viscoelastic fluid by analytical calculations and simulations. Our goal is to characterize the properties of the viscoelastic fluid, which will ultimately be used to study embedded objects. Analytically, we consider the linearized Navier-Stokes equations with a time-dependent relaxation modulus, i.e., an integrodifferential equation for the velocity field. ${ }^{6}$ The relaxation modulus follows from the Rouse model of polymer dynamics, ${ }^{10}$ a special case of the generalized Maxwell model. In simulations, we employ the MPC approach that has successfully been applied to study the structural and dynamical properties of a wide range of polymeric systems. ${ }^{12,27,28,43-51}$ It correctly captures hydrodynamic interactions ${ }^{43,48}$ and can efficiently be parallelized on various platforms, especially on graphics processing units (GPUs). ${ }^{52,53}$

We analyze the fluid properties in terms of velocity autocorrelation functions. An analytical solution for the transverse velocity autocorrelation function is conveniently obtained in Fourier space, with respect to position, and in Laplace space, with respect to time. Inverse Laplace transformation yields a strongly time-dependent transverse autocorrelation function that exhibits damped oscillations. Both the damping and oscillation frequencies depend on the relaxation times of the polymer and the wave vector. Independent of the polymer length, the (transverse) velocity autocorrelation function $C(t)$ exhibits a long-time tail on large length scales, with the time dependence $t^{-3 / 2}$ as is well established for
Newtonian fluids. ${ }^{42,54-60}$ Hence, hydrodynamic correlations determine the dynamical properties of a melt of phantom polymers on large length scales. This is reflected in the polymer center-of-mass diffusion coefficient that exhibits the polymer length dependence according to the hydrodynamic Zimm model. ${ }^{10}$

The article is organized as follows. Section II presents the polymer model and a description of the viscoelastic fluid in terms of a modified Navier-Stokes equation. Velocity autocorrelation functions of the fluid are introduced, and their analytical solutions are presented in Sec. IV. The dynamics of the center-of-mass of an individual (tagged) polymer is discussed in Sec. V. Section III describes the MPC implementation, and Sec. VI presents the simulation results and a comparison with theoretical predictions. Finally, the main results and aspects of our study are summarized in Sec. VII. Appendixes $\mathrm{A}$ and $\mathrm{B}$ describe details of the calculation of inverse Laplace transformations. Appendix $\mathrm{C}$ illustrates the derivation of the center-of-mass velocity autocorrelation function of a tagged polymer.

\section{MODEL OF VISCOELASTIC FLUID}

\section{A. Polymer dynamics}

We consider an ensemble of linear phantom polymers, each composed of $N$ monomers. The bonds between subsequent monomers are described by the harmonic Hamiltonian,

$$
H=\frac{K}{2} \sum_{i=1}^{N-1}\left(\boldsymbol{r}_{i+1}-\boldsymbol{r}_{i}\right)^{2} .
$$

In the stationary state, this leads to a Gaussian partition function capturing the conformational degrees of freedom of the polymer. ${ }^{61}$ The overdamped equation of motion for the position $r_{i}(t)$ of monomer $i$, corresponding to the Rouse description of polymer physics, ${ }^{10,62}$ is then

$$
\dot{\boldsymbol{r}}_{i}=-\frac{1}{\gamma} \frac{\partial H}{\partial \boldsymbol{r}_{i}}+\frac{1}{\gamma} \boldsymbol{\Gamma}_{i} .
$$

Here, $\dot{r}_{i}(t)$ is the monomer velocity at time $t, k_{B}$ is the Boltzmann constant, $T$ is the temperature, $\gamma$ is the friction coefficient, and $\boldsymbol{\Gamma}_{i}$ represent stationary, Markovian, and Gaussian random processes with zero mean and the second moments $(\alpha, \beta \in\{x, y, z\})$,

$$
\left\langle\Gamma_{i \alpha}(t) \Gamma_{j \beta}\left(t^{\prime}\right)\right\rangle=2 k_{B} T \gamma \delta_{i j} \delta_{\alpha \beta} \delta\left(t-t^{\prime}\right) .
$$

The coefficient $K$ in Eq. (1) is related to the mean square bond length $l^{2}$ via $K=3 k_{B} T / l^{2}$.

The solution of Eq. (2) is ${ }^{63,64}$

$$
\boldsymbol{r}_{i}(t)=\sum_{n=0}^{N-1} \chi_{n}(t) b_{n}^{(i)},
$$

with the eigenfunctions

$$
b_{n}^{(i)}=\sqrt{\frac{2}{N}} \cos \left(\frac{n \pi}{N}\left[i-\frac{1}{2}\right]\right)
$$

and 


$$
\boldsymbol{\chi}_{0}(t)=\sqrt{\frac{1}{2 N}} \sum_{i=1}^{N} \boldsymbol{r}_{i}(t)=\sqrt{\frac{N}{2}} \boldsymbol{r}_{c m} .
$$

The correlation functions of the mode amplitudes $\chi_{n}$ are obtained as $(n, m \in[1, N-1])$

$$
\left\langle\chi_{n}(t) \cdot \chi_{m}(t)\right\rangle=\frac{l^{2} \delta_{n m}}{4 \sin ^{2}(n \pi /(2 N))} e^{-t / \tau_{n}},
$$

with the relaxation times

$$
\tau_{n}=\frac{\gamma l^{2}}{12 k_{B} T \sin ^{2}(n \pi / 2 N)} .
$$

In the continuum limit $N \rightarrow \infty, l \rightarrow 0$ such that $L=N l$ remains constant, the well-known expression

$$
\tau_{n}=\frac{\gamma L^{2}}{3 \pi^{2} k_{B} T n^{2}}=\frac{\tau_{R}}{n^{2}}
$$

of the continuous Rouse model is obtained, with the Rouse relaxation time $\tau_{R}=\hat{\gamma} l L^{2} / 3 \pi^{2} k_{B} T$, the friction coefficient $\hat{\gamma}$ per length, and the bond length (Kuhn length) $l=2 l_{p}$, where $l_{p}$ is the persistence length. ${ }^{10,62}$

The current formulation of the model, with $K=3 k_{B} T / l^{2}$, applies to equilibrium systems only and cannot reproduce some nonequilibrium properties, such as shear thinning. To capture such effects, the stretching of polymer bonds by the external forces needs to be prevented. In the case of simple shear, this is easily achieved by a shear-dependent coefficient $\mu(\dot{\gamma})$ and the modified force coefficient $K=3 \mu(\dot{\gamma}) k_{B} T / l^{2}$, where $\dot{\gamma}$ is the shear rate. The coefficient $\mu$ follows from the inextensibility constraint $\sum_{i=1}^{N-1}\left\langle\left(\boldsymbol{r}_{i+1}-\boldsymbol{r}_{i}\right)^{2}\right\rangle=(N-1) l^{2}$. ${ }^{65,66}$ More generally, the constraint $\left\langle\left(\boldsymbol{r}_{i+1}-\boldsymbol{r}_{i}\right)^{2}\right\rangle=l^{2}$ for every bond can be applied with a corresponding number of Lagrangian multipliers. Even for dumbbells, shear thinning is obtained with this length constraint. ${ }^{14}$

\section{B. Modified Navier-Stokes equation}

The viscous properties of Newtonian fluids are described by the Navier-Stokes equations. ${ }^{67}$ In the absence of external forces, the corresponding linearized equation for the fluid momentum is ${ }^{67}$

$$
\varrho \frac{\partial \boldsymbol{v}(\boldsymbol{r}, t)}{\partial t}=-\nabla p+\eta \Delta \boldsymbol{v}(\boldsymbol{r}, t)
$$

with the fluid velocity $\boldsymbol{v}(\boldsymbol{r}, t)$ and pressure $p(\boldsymbol{r}, t)$ fields at the position $r$ and time $t$, the fluid mass density $\varrho$, and the shear viscosity $\eta$. We want to consider a viscoelastic fluid composed of phantom polymers (Sec. II A). Viscoelasticity is incorporated in the Navier-Stokes equation by the (heuristic) extension,

$$
\varrho \frac{\partial \boldsymbol{v}(\boldsymbol{r}, t)}{\partial t}=-\nabla p+\int_{0}^{t} G\left(t-t^{\prime}\right) \Delta \boldsymbol{v}\left(\boldsymbol{r}, t^{\prime}\right) d t^{\prime} .
$$

Here, $G(t)$ is the shear relaxation modulus, which is independent of spatial coordinates and vanishes in the asymptotic limit $\left(t-t^{\prime}\right) \rightarrow \infty$.

The relaxation modulus $G^{p}(t)$ for the phantom polymers of Sec. II A has been determined in Ref. 10 as $(t \geq 0)$

$$
G^{p}(t)=\varphi k_{B} T \sum_{n=1}^{N-1} e^{-2 t / \tau_{n}},
$$

where $\varphi$ is the number of polymers per volume. The latter is related to the mass density $\varrho$ via

$$
\varphi=\frac{\varrho}{m N}=\frac{\phi}{N}
$$

where $m$ is the monomer mass and $\phi$ is the overall monomer concentration. The complete fluid relaxation modulus $G(t)$ is, aside from the polymer-bond contribution, determined by the ideal gas contribution of the individual monomers due to their thermal motion. Hence, we use the relaxation modulus,

$$
G(t)=\eta \delta(t)+\varphi k_{B} T \sum_{n=1}^{N-1} e^{-2 t / \tau_{n}} .
$$

Then, the Navier-Stokes equation (11) reduces to that of a Newtonian fluid in the case of a monomer solution $(N=1)$.

The viscosity $\eta_{f}$ of the viscoelastic fluid follows from $G(t)$ via $^{10}$

$$
\eta_{f}=\int_{0}^{\infty} G(t) d t
$$

which yields, with Eq. (9), ${ }^{10}$

$$
\eta_{f}=\eta+\frac{\varphi k_{B} T}{2} \sum_{n=1}^{N-1} \tau_{n}=\eta+\frac{\varphi \gamma l^{2}\left(N^{2}-1\right)}{36} .
$$

With the density $\varphi$ of Eq. (13), the fluid viscosity becomes

$$
\eta_{f}=\eta+\frac{\phi \gamma l^{2}}{36}\left(N-\frac{1}{N}\right) \text {. }
$$

For long polymers $(N \gg 1)$ and fixed $\phi, \eta_{f}$ is dominated by the bond contribution $\left(G^{P}\right)$ and $\eta$ is negligible. Then, the fluid viscosity increases linearly with the degree of polymerization, $N$.

\section{MESOSCALE HYDRODYNAMICS: MULTIPARTICLE COLLISION DYNAMICS}

The MPC method for the simulation of the polymer dynamics proceeds in two steps-streaming and collision. In the streaming step over a time interval $h$, where $h$ is denoted as collision time, Newton's equations of motion for the monomers,

$$
m \ddot{\boldsymbol{r}}_{i}=-\frac{\partial H}{\partial \boldsymbol{r}_{i}},
$$

are solved by the velocity Verlet algorithm, ${ }^{68}$ with the Hamiltonian of Eq. (1). Since we consider phantom polymers, only bond forces contribute to the monomer dynamics. Other monomer-monomer interactions are implemented via MPC. Here, monomers are sorted into cubic cells of side length $a$, with the cells forming a complete tiling of the simulation volume, defining the collision environment. We apply the Stochastic Rotation Dynamics (SRD) ${ }^{21,22,69}$ version of MPC, ${ }^{23}$ where the relative monomer velocities, with respect to the center-of-mass velocity of all monomers in a collision cell, are rotated around a randomly oriented axis by a fixed angle $\alpha$. This yields the new monomer velocities,

$$
\boldsymbol{v}_{i}(t+h)=\overline{\boldsymbol{v}}_{i}(t+h)+(\mathbf{R}(\alpha)-\mathbf{E})\left(\overline{\boldsymbol{v}}_{i}-\overline{\boldsymbol{v}}_{\mathrm{cm}}^{c}\right),
$$


where $\overline{\boldsymbol{v}}_{i}(t+h)$ is the monomer velocity after streaming, $\mathbf{R}(\alpha)$ is the rotation matrix, $\mathbf{E}$ is the unit matrix, and

$$
\boldsymbol{v}_{\mathrm{cm}}^{c}(t)=\frac{1}{N_{c}} \sum_{j=1}^{N_{c}} \boldsymbol{v}_{j}(t)
$$

is the center-of-mass velocity of the monomers in the cell of particle $i$, with $N_{c}$ being the total number of monomers in that particular cell. The random orientation of the rotation axis is chosen independently for every collision step and every collision cell. Partitioning of the simulation volume into collision cells implies violation of Galilean invariance. To re-establish Galilean invariance, a random shift of the collision lattice is performed at every collision step. ${ }^{23,69}$ MPC conserves mass, momentum, and energy on the collision-cell level, which leads to correlations ${ }^{70,71}$ between the particles and long-range hydrodynamic interactions. ${ }^{60}$ To maintain a constant temperature, the Maxwell-Boltzmann-scaling (MBS) thermostat is applied at every collision step and for every collision cell.

The simulations are performed with the hybrid program OpenMPCD, ${ }^{73}$ a software suite implementing MPC-SRD ${ }^{21-23,74}$ combined with molecular dynamics simulations (MD) (velocity Verlet algorithm ${ }^{68}$ ). Both the MPC and the MD part of the polymer dynamics-only phantom polymers with intramolecular bond interactions are considered-are executed in a massively parallel manner on a GPU (double precision). The program exhibits excellent performance on graphical processing units (GPUs) ${ }^{52}$ supporting the $\mathrm{CUDA}^{75}$ programming framework, such as NVIDIA Tesla accelerators.

Dimensionless units are introduced by scaling length by the cell size $a$, energy by $k_{B} T$, and time by $\sqrt{m a^{2} / k_{B} T}$. This corresponds to the choice $a=k_{B} T=m=1$. We choose the collision time $h=0.1 \sqrt{m a^{2} / k_{B} T}$, the rotation angle $\alpha=2.27 \mathrm{rad} \approx 130^{\circ}$, and the mean number of monomers in a collision cell $\left\langle N_{c}\right\rangle=10$. The latter is equivalent to the mean fluid density $\varrho=10 \mathrm{~m} / \mathrm{a}^{3}$. A MD time step of $\Delta t=0.02 \sqrt{m a^{2} / k_{B} T}$, smaller than the collision time step, is used in order to resolve the polymer dynamics adequately. Threedimensional periodic systems are considered with a cubic simulation box of side length $L_{S}=30 a$, if not indicated otherwise, corresponding to a total number of $N_{t o t}=2.7 \times 10^{5}$ monomers $/$ MPC particle. Simulations of a monomer fluid, i.e., a bare MPC fluid, yield the viscosity $\eta / \sqrt{m k_{B} T / a^{4}}=8.7 .^{76}$ In the following, the units $a, k_{B} T$, and $m$ will be dropped, i.e., are set to unity. For the results presented in Sec. VI, between $2 \times 10^{7}$ and $1 \times 10^{8}$ MPC steps have been performed, typically approximately $4 \times 10^{7}$.

Simulations are initialized by placing the first monomer of every polymer at a random point in the simulation volume sampled from a uniform distribution. Subsequent bound monomers are placed by choosing a randomly oriented unit bond vector. Initial velocities of each monomer are assigned independently with Cartesian components taken from a standard normal distribution.

\section{VELOCITY CORRELATION FUNCTION OF VISCOELASTIC FLUID}

The linear equation (11) can be solved using Fourier and Laplace transforms. Spatial Fourier transformation (denoted by a tilde) of the velocity,

$$
\tilde{\boldsymbol{v}}(\boldsymbol{k}, t)=\int \boldsymbol{v}(\boldsymbol{r}, t) e^{-i \boldsymbol{k} \cdot \boldsymbol{r}} d^{3} r,
$$

where $\tilde{\boldsymbol{v}}(\boldsymbol{k}, t)$ denotes the transformed velocity, yields

$$
\varrho \frac{\partial \tilde{\boldsymbol{v}}(\boldsymbol{k}, t)}{\partial t}=-i \boldsymbol{k} \tilde{p}(\boldsymbol{k}, t)-\boldsymbol{k}^{2} \int_{0}^{t} G\left(t-t^{\prime}\right) \tilde{\boldsymbol{v}}\left(\boldsymbol{k}, t^{\prime}\right) d t^{\prime}
$$

By multiplying this equation with $\tilde{\boldsymbol{v}}(-\boldsymbol{k}, 0)$, we obtain

$$
\varrho \frac{\partial \tilde{C}^{T}(\boldsymbol{k}, t)}{\partial t}=-\boldsymbol{k}^{2} \int_{0}^{t} G\left(t-t^{\prime}\right) \tilde{C}^{T}\left(\boldsymbol{k}, t^{\prime}\right) d t^{\prime}
$$

for the transverse velocity autocorrelation function,

$$
\tilde{C}^{T}(\boldsymbol{k}, t)=\left\langle\tilde{\boldsymbol{v}}^{T}(\boldsymbol{k}, t) \cdot \tilde{\boldsymbol{v}}^{T}(-\boldsymbol{k}, 0)\right\rangle,
$$

where the brackets denote statistical averaging. The transverse component $\tilde{\boldsymbol{v}}^{T}(\boldsymbol{k}, t)$ is the component of the Fourier-space velocity $\tilde{\boldsymbol{v}}(\boldsymbol{k}, t)=\tilde{\boldsymbol{v}}^{L}(\boldsymbol{k}, t)+\tilde{\boldsymbol{v}}^{T}(\boldsymbol{k}, t)$ that is perpendicular to the Fourier vector $\boldsymbol{k}$, i.e., $\boldsymbol{k} \cdot \tilde{\boldsymbol{v}}^{T}(\boldsymbol{k}, t)=0$. Laplace transformation (denoted by a circumflex) with respect to time,

$$
\hat{C}^{T}(\boldsymbol{k}, s)=\int_{0}^{\infty} \tilde{C}^{T}(\boldsymbol{k}, t) e^{-s t} d t,
$$

yields

$$
\hat{C}^{T}(\boldsymbol{k}, s)=\frac{\varrho \tilde{C}^{T}(\boldsymbol{k}, 0)}{\varrho s+\boldsymbol{k}^{2} \hat{G}(s)} .
$$

We assume that the system is in thermal equilibrium at $t=0$, hence, ${ }^{60}$

$$
\tilde{C}^{T}(\boldsymbol{k}, 0) \equiv \tilde{C}^{T}(0)=\frac{2 k_{B} T}{\varrho} .
$$

The Laplace transform of $G(t)$, Eq. (14), is ${ }^{77,78}$

$$
\hat{G}(s)=\eta+\varphi k_{B} T \sum_{n=1}^{N-1} \frac{1}{s+2 / \tau_{n}},
$$

and we thus obtain

$$
\hat{C}^{T}(\boldsymbol{k}, s)=\frac{\varrho \tilde{C}^{T}(0)}{\varrho s+\boldsymbol{k}^{2}\left(\eta+\varphi k_{B} T \sum_{n=1}^{N-1} \frac{1}{s+2 / \tau_{n}}\right)} .
$$

The explicit expression $\tilde{C}^{T}(\boldsymbol{k}, t)$ for the inverse Laplace transform of this function is presented in Eq. (A4) of Appendix A.

The velocity correlation function $C(t)=\langle\boldsymbol{v}(\boldsymbol{r}, t) \cdot \boldsymbol{v}(\boldsymbol{r}, 0)\rangle$ follows by inverse Fourier and Laplace transformation. To eliminate the spatial dependence, we average the correlation function with the distribution function for $\boldsymbol{r}(t) .^{55,57,60}$ Adopting the Lagrangian description of the fluid, where a fluid element is followed as it moves through space and time, we obtain, in general,

$$
\begin{aligned}
C(t) & =\frac{1}{(2 \pi)^{3}} \int \tilde{C}(\boldsymbol{k}, t)\left\langle e^{i \boldsymbol{k} \cdot(\boldsymbol{r}(t)-\boldsymbol{r}(0))}\right\rangle d^{3} k \\
& =\frac{1}{(2 \pi)^{3}} \int \tilde{C}(\boldsymbol{k}, t) e^{-\boldsymbol{k}^{2}\left\langle(r(t)-\boldsymbol{r}(0))^{2}\right\rangle / 6} d^{3} k
\end{aligned}
$$

due to the Gaussian nature of the displacement distribution function. ${ }^{10}$ The mean square displacement (MSD), averaged over all monomers, is ${ }^{10}$ 


$$
\left\langle\Delta \boldsymbol{r}(t)^{2}\right\rangle=\left\langle(\boldsymbol{r}(t)-\boldsymbol{r}(0))^{2}\right\rangle=6 D_{\mathrm{cm}} t+\left\langle\Delta \boldsymbol{r}(t)_{m}^{2}\right\rangle,
$$

where $D_{\mathrm{cm}}=k_{B} T / \gamma N$ is the center-of-mass diffusion coefficient, and with the results of Sec. II A, we obtain the average monomer MSD in the polymer center-of-mass reference frame,

$$
\left\langle\Delta \boldsymbol{r}(t)_{m}^{2}\right\rangle=\frac{l^{2}}{2 N} \sum_{n=1}^{N-1} \frac{1}{\sin ^{2}(n \pi /(2 N))}\left(1-e^{-t / \tau_{n}}\right) .
$$

Examples of the correlation function $C(t)$ for various polymer lengths are discussed in Secs. IV A-IV D.

\section{A. Newtonian fluid $(N=1)$}

A Newtonian fluid is recovered for $N=1$ and correspondingly $G(t)=\eta \delta(t)$. The inverse Laplace transformation of

$$
\hat{C}^{T}(\boldsymbol{k}, s)=\frac{\tilde{C}^{T}(0)}{s+\boldsymbol{k}^{2} v},
$$

with the kinematic viscosity $v=\eta / \varrho$, yields the time-dependent velocity-correlation function in Fourier space,

$$
\tilde{C}^{T}(\boldsymbol{k}, t)=\tilde{C}^{T}(0) e^{-v \boldsymbol{k}^{2} t},
$$

in agreement with previous studies. ${ }^{60}$ With Eq. (27), the correlation function $C(t)=C^{L}(t)+C^{T}(t)$ of Eq. (30) becomes, in the long-time limit, ${ }^{54-57,60}$

$$
C(t) \approx C^{T}(t)=\frac{k_{B} T}{4 \varrho} \frac{1}{\left(\pi\left[v+D_{\mathrm{cm}}\right] t\right)^{3 / 2}},
$$

since the contribution of the longitudinal velocity correlation, $C^{L}(t)$, decays exponentially. ${ }^{48,60}$

\section{B. Dumbbell fluid $(\boldsymbol{N}=\mathbf{2})$}

Polymer-like aspects are already captured by a dumbbell (dimer)-i.e., two bound monomers-, at least as long as the longest relaxation time of a polymer dominates its internal dynamics. Here, Eq. (A4) assumes the form

$$
\tilde{C}^{T}(\boldsymbol{k}, t)=\frac{\tilde{C}^{T}(\boldsymbol{k}, 0)}{\omega} e^{-\zeta t}\left(\left[\frac{2}{\tau_{1}}-\zeta\right] \sin (\omega t)+\omega \cos (\omega t)\right),
$$

with the abbreviations

$$
\begin{aligned}
& \zeta=\frac{1}{2}\left(\frac{2}{\tau_{1}}+\boldsymbol{k}^{2} v\right), \\
& \omega=\frac{1}{\tau_{1}} \sqrt{2 \boldsymbol{k}^{2} \tau_{1}\left(v_{f}-v\right)-\frac{1}{8}\left(\boldsymbol{k}^{2} v \tau_{1}-2\right)^{2}},
\end{aligned}
$$

and the kinematic viscosity $v_{f}=\eta_{f} / Q=v+\varphi k_{B} T \tau_{1} /(2 Q)>v$. The correlation function (36) exhibits exponentially damped oscillations, where both the frequency, $\omega$, and the damping, $\zeta$, depend on the relaxation time $\tau_{1}$.

Evidently, the radicand in Eq. (38) is always negative for $v=v_{f}$. More generally, in the case of a negative radicand, the substitution $\omega=i \lambda$, with

$$
\lambda=\frac{1}{\tau_{1}} \sqrt{-2 \boldsymbol{k}^{2} \tau_{1}\left(v_{f}-v\right)+\frac{1}{8}\left(\boldsymbol{k}^{2} v \tau_{1}-2\right)^{2}},
$$

yields the correlation function

$$
\tilde{C}^{T}(k, t)=\frac{\tilde{C}^{T}(\boldsymbol{k}, 0)}{\lambda} e^{-\zeta t}\left(\left[\frac{2}{\tau_{1}}-\zeta\right] \sinh (\lambda t)+\lambda \cosh (\lambda t)\right) .
$$

Since $\zeta>\lambda$, we obtain a nonoscillating correlation function. Equation (38) or Eq. (39) clearly reveal a qualitative different dynamical behavior due to polymer elasticity (viscoelasticity). An oscillatory correlation function appears for $v_{f}>v$ only. There are two obvious limits with only exponentially decaying correlation functions, namely, $|\boldsymbol{k}| \rightarrow 0$ and $|\boldsymbol{k}| \rightarrow \infty$, which correspond to large and small scales, respectively.

In the limit $\lambda t \gg 1$, Eq. (40) becomes

$$
\tilde{C}^{T}(\boldsymbol{k}, t)=\tilde{C}^{T}(\boldsymbol{k}, 0)\left(\frac{1}{\lambda \tau_{1}}-\frac{\zeta}{2 \lambda}+\frac{1}{2}\right) e^{-(\zeta-\lambda) t} .
$$

For $|\boldsymbol{k}| \rightarrow 0$, the difference in the exponent reduces to $\zeta-\lambda=k^{2}(v+$ $\left.\varphi k_{B} T \tau_{1} / 2 \varrho\right)+O\left(k^{4}\right)$ [Eq. (16)], and the correlation function decays exponentially with the total kinematic viscosity, $v_{f}$,

$$
\tilde{C}^{T}(\boldsymbol{k}, t)=\tilde{C}^{T}(\boldsymbol{k}, 0) e^{-v_{f} \boldsymbol{k}^{2} t} .
$$

Then, Fourier transformation with respect to $k$ of Eq. (42) yields a long-time tail $C^{T}(t) \sim\left(v_{f} t\right)^{-3 / 2}$ on large length and long time scales. Conversely, on small length scales $|\boldsymbol{k}| \rightarrow \infty$, the exponent becomes $\zeta-\lambda=2\left(1+v_{f} / v\right) / \tau_{1}+O\left(1 / \boldsymbol{k}^{2}\right)$ and the decay of the correlation function depends only weakly on the wave vector. In both cases, the decline of $\tilde{C}^{T}(\boldsymbol{k}, t)$ is determined by the properties of the dumbbell rather than the individual monomers.

Figure 1 provides examples of the correlation function $\tilde{C}^{T}(\boldsymbol{k}, t)$ for various wave vectors and a specific set of parameters (see the figure caption). Note that the oscillating correlation functions assume positive and negative values. The correlation functions for the smallest $(k=0.09)$ and the largest $(k=2)$ displayed $\boldsymbol{k}$ vector decay

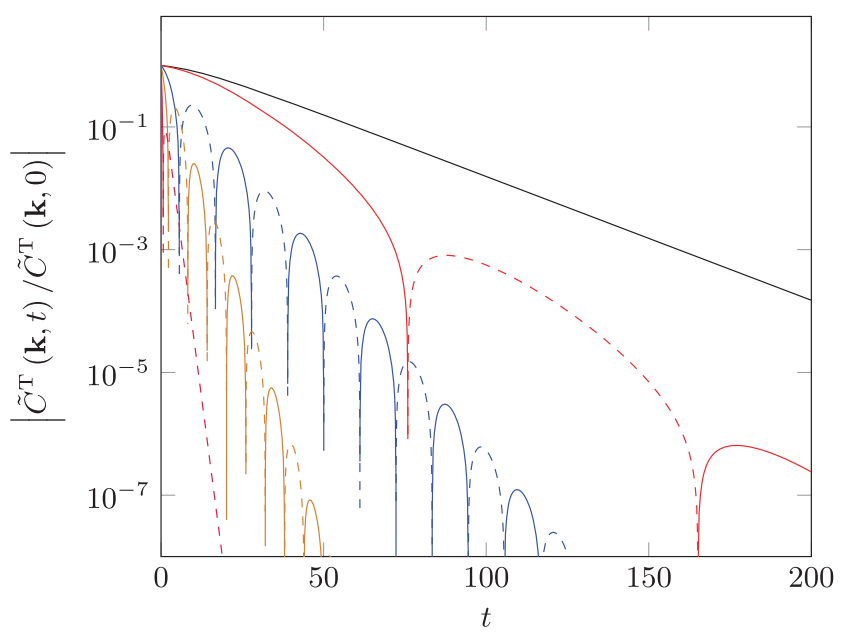

FIG. 1. Transverse velocity autocorrelation function $\tilde{C}^{T}(\boldsymbol{k}, t) / \tilde{C}^{T}(\boldsymbol{k}, 0)$ of dumbbells $[\mathrm{Eq}$. (36) or (40)] as a function of time for the wave vectors $|\boldsymbol{k}|=0.09,0.11$, $0.4,0.8$, and 2 (top to bottom). The other parameters are $\eta=8.7, \varrho=10, \varphi k_{B} T=5$, and $\tau_{1}=13.4$. Due to viscoelasticity, the correlation function oscillates for certain wave vectors assuming positive (solid) and negative (dashed) values. 
exponentially according to Eq. (41), whereas those for in-between $\boldsymbol{k}$ values exhibit exponentially damped oscillations, Eq. (36). In the latter case, the oscillation frequency increases with increasing wave number. The decay rates in the limit $|\boldsymbol{k}| \rightarrow 0$ and $|\boldsymbol{k}| \rightarrow \infty$ agree with the values discussed above.

\section{Continuous polymer $(N \rightarrow \infty)$}

In the case of continuous polymers, $N \rightarrow \infty$, the relaxation modulus (14) becomes

$$
G(t)=\eta \delta(t)+\varphi k_{B} T \sum_{n=1}^{\infty} e^{-2 t n^{2} / \tau_{R}}
$$

Two limiting cases can be distinguished:

(i) $t / \tau_{R} \gg 1-$ The sum in Eq. (43) is dominated by the mode $n=1$, corresponding to the dumbbell considered in Sec. IV B with the relaxation time $\tau_{1}=\tau_{R}$. Again, on large length scales, the velocity correlation function decays exponentially with the viscosity $\eta_{f}=\eta+\varphi k_{B} T \tau_{R} / 2$, where $\eta$ can be neglected for long polymers due to the large Rouse time.

(ii) $t / \tau_{R} \ll 1-$ The sum of modes in Eq. (43) can be replaced by an integral over $n,,^{10,79}$ and we straightforwardly obtain

$$
G(t)=\eta \delta(t)+\frac{\varphi k_{B} T}{2} \sqrt{\frac{\pi \tau_{R}}{2 t}}
$$

Laplace transformation yields

$$
\hat{G}(s)=\eta+\frac{\varphi k_{B} T \pi}{2} \sqrt{\frac{\tau_{R}}{2 s}},
$$

and the velocity correlation function becomes

$$
\hat{C}^{T}(\boldsymbol{k}, s)=\frac{\varrho \tilde{C}^{T}(\boldsymbol{k}, 0)}{\varrho s+\boldsymbol{k}^{2}\left(\eta+\frac{\varphi k_{B} T \pi}{2} \sqrt{\frac{\tau_{R}}{2 s}}\right)} .
$$

Inverse Laplace transformation (cf. Appendix B) yields, neglecting $\eta$

$$
\tilde{C}^{T}(\boldsymbol{k}, t)=\tilde{C}^{T}(0) f\left(\left[\boldsymbol{k}^{2} \varphi k_{B} T \pi \sqrt{\tau_{R}} /(2 \sqrt{2} \varrho)\right]^{2 / 3} t\right),
$$

with the function $f(x)$ specified in Eq. (B5). Hence, $\tilde{C}^{T}(\boldsymbol{k}, t)$ scales with $\left(k^{4 / 3} t\right)$ as already pointed out in Ref. 42 . find

In the asymptotic limit of a large argument of $f$ in Eq. (47), we

$$
\tilde{C}^{T}(\boldsymbol{k}, t)=-\frac{\sqrt{2} \varrho \tilde{C}^{T}(\boldsymbol{k}, 0)}{\varphi k_{B} T \pi \sqrt{\pi \tau_{R}}} \frac{1}{\boldsymbol{k}^{2} t^{3 / 2}} .
$$

Note that the correlation function is negative. The correlation function exhibits a long-time-tail-type time dependence $t^{-3 / 2}$ that is very different from the exponential function of Eq. (34) for individual monomers. Polymer elasticity completely changes the time and wave-vector dependence of the correlation function. However, as shown in Sec. V A 2, this does not affect the long-time-type decay of the correlation function in real space.
Inserting $\tilde{C}^{T}(\boldsymbol{k}, 0)$ of Eq. (27) and the polymer concentration of Eq. (13) into Eq. (48), we find

$$
\tilde{C}^{T}(\boldsymbol{k}, t)=-\frac{2 \sqrt{2}}{\pi^{3 / 2} \phi l \sqrt{\tau_{l}}} \frac{1}{\boldsymbol{k}^{2} t^{3 / 2}},
$$

with the abbreviation $\tau_{l}=\hat{\gamma} l /\left(3 \pi^{2} k_{B} T\right)$. Thus, the fluid correlation function $\tilde{C}(\boldsymbol{k}, t)$ is independent of the polymer length in the time interval $\tau_{l} \ll t \ll \tau_{R}$ for long polymers $(L / l \gg 1)$; it depends on the overall monomer density only.

\section{Asymptotic behavior for $t \rightarrow \infty$}

The asymptotic time dependence of the correlation function $C^{T}(t)$ for $t \rightarrow \infty$ follows from Eq. (29) in the limit $s \rightarrow 0$. Neglecting $s$ in the sum over modes in Eq. (29), the correlation function reduces to $\hat{C}^{T}(\boldsymbol{k}, s)=\tilde{C}^{T}(0) /\left(s+\boldsymbol{k}^{2} v_{f}\right)$, with the total kinematic viscosity $v_{f}=\eta_{f} / \varrho$. Then, Fourier and Laplace transformations yield

$$
C^{T}(t)=\frac{\tilde{C}^{T}(0)}{8\left(\pi v_{f} t\right)^{3 / 2}},
$$

independent of the polymer length. This result is consistent with the limiting cases discussed in Secs. IV A and IV C and corresponds to the long-time tail of simple fluids. ${ }^{54-57,60}$ Hence, on large length scales and for long times, the polymer melt of phantom chains exhibits fluid-like behavior similar to simple fluids, but with the total viscosity $\eta_{f}$ determined by polymer elasticity.

\section{E. Oseen tensor-type behavior} yields

Integration of the correlation function $\tilde{C}^{T}(\boldsymbol{k}, t)$ over time

$$
T(\boldsymbol{k})=\int_{0}^{\infty} \tilde{C}^{T}(\boldsymbol{k}, t) d t=\hat{C}^{T}(\boldsymbol{k}, 0)=\frac{\varrho \tilde{C}^{T}(0)}{\boldsymbol{k}^{2} \eta_{f}},
$$

with the use of the definition of the Laplace transform (25) and Eq. (29). Hence, $T(\boldsymbol{k}) \sim 1 / \boldsymbol{k}^{2}$, similar to the Oseen tensor of a Newtonian fluid, ${ }^{7,10,60}$ but with the viscosity of the polymeric fluid. Fourier transformation with respect to $\boldsymbol{k}$ leads then to a long-range interaction $\sim 1 /|\boldsymbol{r}|$ in three-dimensional space. As a consequence, the considered viscoelastic phantom polymer melt exhibits the properties of Newtonian fluids in terms of long-range fluid interactions.

\section{CENTER-OF-MASS DYNAMICS OF INDIVIDUAL POLYMERS}

The equations of motion (2) describe the dynamics of an isolated polymer exposed to thermal noise. We now consider an individual polymer embedded in other, identical polymers, accounting for the environment by including the convective transport velocity following from Eq. (11). Hence, we set

$$
\dot{\boldsymbol{r}}_{i}(t)=\boldsymbol{v}\left(\boldsymbol{r}_{i}, t\right)-\frac{1}{\gamma} \frac{\partial H}{\partial \boldsymbol{r}_{i}}+\frac{1}{\gamma} \boldsymbol{\Gamma}_{i}(t),
$$

with the fluid velocity $\boldsymbol{v}\left(\boldsymbol{r}_{i}, t\right)$ at the location of monomer $i$. Considering the center-of-mass motion only, we find

$$
\dot{\boldsymbol{r}}_{\mathrm{cm}}(t)=\frac{1}{N} \sum_{i=1}^{N} \boldsymbol{v}\left(\boldsymbol{r}_{i}, t\right)+\frac{1}{\gamma N} \sum_{i=1}^{N} \boldsymbol{\Gamma}_{i}(t)
$$


for the position $\boldsymbol{r}_{\mathrm{cm}}$ and velocity $\dot{\boldsymbol{r}}_{\mathrm{cm}}$ of the center of mass of a particular polymer.

\section{A. Center-of-mass velocity correlation function} by $(t>0)$

The center-of-mass velocity autocorrelation function is given

$$
\begin{aligned}
C_{\mathrm{cm}}(t) & =\left\langle\dot{\boldsymbol{r}}_{\mathrm{cm}}(t) \cdot \dot{\boldsymbol{r}}_{\mathrm{cm}}(0)\right\rangle \\
& =\frac{1}{N^{2}} \sum_{i=1}^{N} \sum_{j=1}^{N}\left\langle\boldsymbol{v}\left(\boldsymbol{r}_{i}, t\right) \cdot \boldsymbol{v}\left(\boldsymbol{r}_{j}, 0\right)\right\rangle .
\end{aligned}
$$

Focusing on the transverse velocity correlation function, in Fourier representation, we obtain ${ }^{42,48}$ (cf. Appendix C for details)

$$
C_{\mathrm{cm}}^{T}(t)=\frac{1}{(2 \pi)^{3} N} \int S(\boldsymbol{k}, t) \tilde{C}^{T}(\boldsymbol{k}, t) d^{3} k,
$$

with $\tilde{C}^{T}(\boldsymbol{k}, t)$ presented in Sec. IV and the dynamic structure factor $^{10,80}$

$$
\begin{aligned}
S(\boldsymbol{k}, t) & =\frac{1}{N} \sum_{i=1}^{N} \sum_{j=1}^{N}\left\langle e^{i \boldsymbol{k} \cdot\left(\boldsymbol{r}_{i}(t)-\boldsymbol{r}_{j}(0)\right)}\right\rangle \\
& =\frac{1}{N} \sum_{i=1}^{N} \sum_{j=1}^{N} \exp \left(-\boldsymbol{k}^{2}\left\langle\left(\boldsymbol{r}_{i}(t)-\boldsymbol{r}_{j}(0)\right)^{2}\right\rangle / 6\right)
\end{aligned}
$$

of the polymer. Note that the solution of the polymer dynamics of Sec. II A yields a Gaussian distribution of the monomer-monomer separation $\boldsymbol{r}_{i}-\boldsymbol{r}_{j}$, with $\left\langle\left(\boldsymbol{r}_{i}-\boldsymbol{r}_{j}\right)^{2}\right\rangle=\left.|i-j|\right|^{2}$, and the mean square displacement

$\left\langle\left(\boldsymbol{r}_{i}(t)-\boldsymbol{r}_{j}(0)\right)^{2}\right\rangle=|i-j| l^{2}+6 D_{c m} t+\frac{6 k_{B} T}{\gamma} \sum_{n=1}^{N-1} \tau_{n} b_{n}^{(i)} b_{n}^{(j)}\left(1-e^{-t / \tau_{n}}\right)$.

Strictly speaking, the mean square displacement has to be obtained from the solution of Eq. (52), which includes the convective flow field induced by neighboring polymers. However, on large length scales and for long polymers, the most significant contribution comes from small $\boldsymbol{k}$ values. Hence, the time dependence of the dynamic structure factor can be neglected and the static structure factor, $S(\boldsymbol{k}, 0)$, can be used, i.e., the relevant properties of the tracer polymer are captured by its equilibrium structure. In general, the term $D_{c m} t$ can be neglected for long polymers because the kinematic viscosity is typically much larger than $D_{c m}$ [cf. Eq. (35)]. Our calculations confirm that these approximations apply and that $C_{c m}(t)$ is essentially identical when using either $S(\boldsymbol{k}, t)$ or $S(\boldsymbol{k}, 0)$, even for dumbbells. Consequently, the time dependence of $C_{c m}(t)$ is completely determined by the correlation function, $\tilde{C}(\boldsymbol{k}, t)$, of the viscoelastic fluid.

\section{Dumbbell fluid $(N=2)$}

For a dumbbell, the dynamics structure factor (56) reads

$$
\begin{aligned}
S(\boldsymbol{k}, t)= & e^{-D_{c m} \boldsymbol{k}^{2} t}\left[\exp \left(-\frac{\boldsymbol{k}^{2} l^{2}}{12}\left(1-e^{-t / \tau_{1}}\right)\right)\right. \\
& \left.+\exp \left(\frac{\boldsymbol{k}^{2} l^{2}}{12}\left(1-e^{-t / \tau_{1}}\right)\right) e^{-\boldsymbol{k}^{2} l^{2} / 6}\right] .
\end{aligned}
$$

The correlation function $C_{\mathrm{cm}}^{T}(t)$ is then obtained by evaluation of Eq. (55) with the correlation function $\tilde{C}^{T}(\boldsymbol{k}, t)$ of Sec. IV B.

For a dumbbell, the contribution of the convective velocity in Eq. (52) to dynamic properties, e.g., an effective relaxation time, is negligible as shown in Ref. 14 and the relaxation time of the Rouse model can be used. As pointed out above, the numerical evaluation of Eq. (55) yields essentially the same correlation function when using either $S(\boldsymbol{k}, t)$ or $S(\boldsymbol{k}, 0)$.

\section{Continuous polymer $(N \rightarrow \infty)$}

In the limit of a continuous polymer, integration of Eq. (55) with the correlation function (49) yields

$$
C_{\mathrm{cm}}^{T}(t)=-\frac{8}{\sqrt{3 l^{3}} \pi^{3} \phi \sqrt{\tau_{l}}} \frac{1}{t^{3 / 2} \sqrt{L}}
$$

for the time interval $\tau_{l} \ll t \ll \tau_{R}$. We take only the static structure factor into account in deriving Eq. (59), i.e., we set $t=0$ in Eq. (57). As our numerical studies show, a more precise account of $S(k, t)$ changes the very short-time behavior of the correlation function but does not affect the longer-time decay, which is of primary interest here.

Evidently, the correlation function (59) exhibits a powerlaw decay $t^{-3 / 2}$ reminiscent to the long-time tail of hydrodynamics. ${ }^{54-57,60}$ However, the asymptotic time regime for $t \rightarrow \infty$, corresponding to the long-time-tail hydrodynamics of simple fluids, is described by Eq. (50). The correlation function (59) emerges from the polymer character of the fluid, with its nearly continuous mode spectrum. The coupling of internal polymer dynamics leads to fluidlike large-scale and long-time correlations. Similar dependencies on time and polymer length, $1 / \sqrt{L}$, have been obtained in Ref. 42 .

\section{B. Center-of-mass diffusion}

The center-of-mass diffusion coefficient follows from the center-of-mass correlation function via the relation

$$
D=\frac{1}{3} \int_{0}^{\infty} C_{\mathrm{cm}}^{T}(t) d t=\frac{1}{3(2 \pi)^{3} N} \int S(\boldsymbol{k}) \hat{C}^{T}(\boldsymbol{k}, 0) d^{3} k
$$

because the integral over the longitudinal contribution of the correlation function vanishes. ${ }^{48,60}$ Evaluation of the integral with Eqs. (27) and (29) yields

$$
D=\frac{8 k_{B} T}{3 \sqrt{6 \pi^{3}} \eta_{f}} \frac{1}{\sqrt{l L}},
$$

for a continuous polymer. This is the diffusion coefficient of a polymer in a solution of viscosity $\eta_{f}$ (Zimm model). ${ }^{10}$ Thus, our phantom-polymer melt yields the same polymer length dependence, i.e., $1 / \sqrt{l L}$, as a polymer in solution. This emphasizes that hydrodynamic interactions are fully developed and determine the diffusive behavior.

\section{SIMULATION RESULTS, COMPARISON WITH THEORY}

\section{A. Correlation function $\tilde{C}^{T}(\boldsymbol{k}, t)$}

In simulations, periodic boundary conditions are applied and the monomer velocities in Fourier space are calculated as 


$$
\tilde{\boldsymbol{v}}(\boldsymbol{k}, t)=\frac{1}{N_{\text {tot }}} \sum_{i=1}^{N_{\text {tot }}} \boldsymbol{v}_{i}(t) e^{-i \boldsymbol{k} \cdot \boldsymbol{r}_{i}(t)} .
$$

Due to the periodic boundary conditions, the Cartesian components $k_{\alpha}$ of the wave vector $\boldsymbol{k}=\left(k_{x}, k_{y}, k_{z}\right)^{T}$ assume the values $k_{\alpha}=2 \pi n_{\alpha} / L$, with $n_{\alpha} \in \mathbb{Z}, \alpha \in\{x, y, z\}$, and $N_{\text {tot }}=N N_{p}$ being the total number of monomers. Note that only $k$-values with $|\boldsymbol{k}| \neq 0$ are allowed. Here, the Fourier transformation [Eq. (21)] of the viscoelastic continuum is adjusted to periodic boundary conditions as described in Ref. 60. In agreement with the results of Ref. 60, the transverse velocity correlation function of the bare MPC fluid (monomers) decays exponentially according to Eq. (34) with the kinematic viscosity $v=0.87$.

\section{Dumbbell fluid $(N=2)$}

Results for the transverse velocity autocorrelation function of dumbbells of various bond lengths are displayed in Fig. 2. We like to mention that accurate simulation data for long times are rather demanding in terms of simulation time, both for viscous and viscoelastic fluids. The correlation functions typically decay over several orders of magnitude in the considered time range, ${ }^{60}$ and the MPC-intrinsic hydrodynamic fluctuations need to be averaged out. Nevertheless, good agreement is obtained between theory and simulations.

The qualitative different behavior in Figs. 2(a) and 2(b) is in agreement with the theoretical expectations discussed in Sec. IV B, since the radicand in Eq. (39) is positive for $l=1$ and negative for $l=\sqrt{3}$. Hence, for $l=1$, the correlation function decays exponentially according to Eq. (40), whereas oscillations occur for longer bonds corresponding to Eq. (36). By fitting the theoretical expressions (36) and (40), respectively, we find the relaxation time $\tau_{1} \approx 2.8 l^{2}$ (see also Ref. 14). This value agrees reasonably well with the theoretical prediction $3.1 l^{2}$ following from the relaxation time [Eq. (8)] with the friction coefficient $\gamma=6 \pi \eta R_{H}$, where the hydrodynamic radius of a monomer is $R_{H}=0.113$.

Evidently, our simulations and the theoretical approach yield long-range hydrodynamic correlations. The emergence of such correlations is not unexpected, since both the MPC simulations and the (generalized) Navier-Stokes equations conserve momentum. For the relatively short polymer chains, Rouse-like relaxation can be expected because Zimm-type hydrodynamics requires long polymers, while the dumbbell relaxation time is only weakly affected by "fluid" correlations. ${ }^{14}$

\section{Decamer fluid $(\mathbf{N}=10)$}

Figure 3 presents simulation and theoretical results of $\tilde{C}^{T}(\boldsymbol{k}, t)$ for decamers of various bond lengths. Fitting of Eq. (A6) to the simulations data yields the relation $\tau_{1} \approx 54 l^{2}$ for the bond-length dependence of the longest relaxation time. The theoretically predicted value $\tau_{1} \approx 63 l^{2}$, according to Eq. (8), is somewhat larger, when the hydrodynamic radius $R_{H}=0.113$ is used. ${ }^{14}$ The relaxation times are, compared to a dumbbell fluid, longer, and only damped oscillating correlation functions occur for the considered $\boldsymbol{k}$ vectors, as expected theoretically. The comparison of Figs. 3(a) and 3(b) indicates an increase in the frequency with increasing relaxation time, which is in agreement with the theoretical expectations. Moreover,
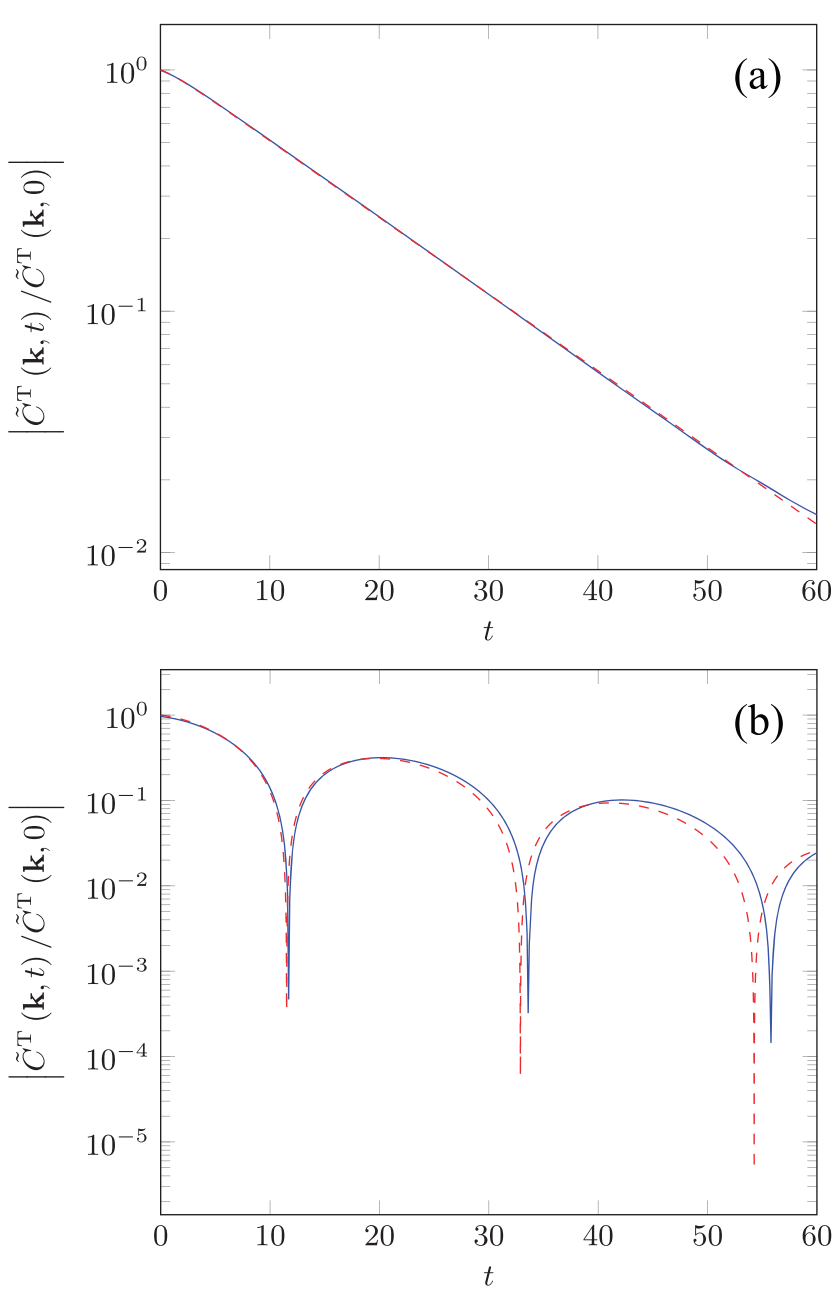

FIG. 2. Transverse velocity autocorrelation functions of dumbbells, i.e., $N=2$, from simulations (solid) and analytical theory (dashed), Eqs. (36) and (40), as functions of time $t$ for the bond length $(a) l=1(K=3)$ and (b) $l=\sqrt{10}(K=0.3)$. The wave vector is, in all cases, $\boldsymbol{k}=(2 \pi / 30,0,0)^{T}$. As in Fig. 1, the correlation function in (b) assumes negative values (even loops). The theoretical curves are fitted to the simulation data.

the evidently different time intervals between zeros of $\tilde{C}^{T}(\boldsymbol{k}, t)$ in Fig. 3(b) reflect the presence of multiple relaxation times.

As for the dumbbell fluid, we find very good agreement between the simulation data and the theoretical prediction over the presented time window. In general, our results emphasize the strongly correlated polymer dynamics by the momentum-conserving interaction, i.e., long-range hydrodynamics.

\section{B. Center-of-mass velocity correlation function in real space}

The autocorrelation function of the center-of-mass velocity of a polymer in real space, Eq. (54), can be directly calculated. For a compressible fluid, such as the MPC fluid, $C_{c m}(t)$ comprises contributions from transverse and longitudinal modes, which 

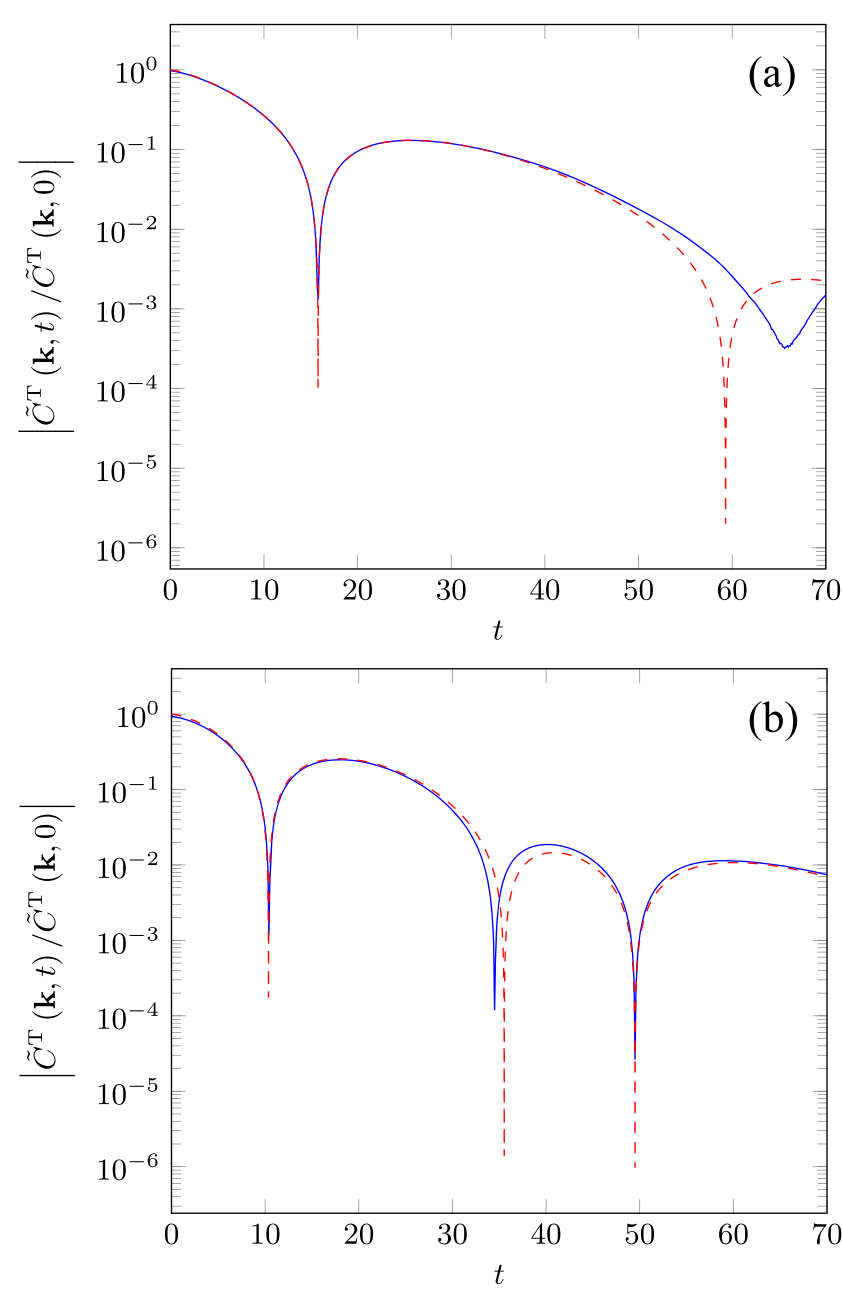

FIG. 3. Transverse velocity autocorrelation functions of decamers $(N=10)$ as a function of time $t$ from simulations (solid) and analytical theory (dashed) for the bond lengths, (a) $l=1(K=3)$ and (b) $l=\sqrt{3}(K=1)$. The wave vector is $\boldsymbol{k}=(2 \pi / 30,0,0)^{\top}$. As in Fig. 1, the correlation functions assume negative values (even loops). The theoretical curves are fitted to the simulation data.

cannot be simply extracted from the correlation function (54) determined in simulations. However, the longitudinal modes affect the short-time behavior of $C_{c m}(t)$ only, since the longitudinal correlation function decays exponentially ${ }^{48,60}$ and the longer-time hydrodynamic properties are determined by the transverse correlation function with its long-time tail. Hence, the correlation function $C_{c m}(t)$ of the MPC fluid exhibits the correct long-time behavior. Moreover, the short-time behavior of the correlation function reflects the partitioning of space into collision cells of the MPC approach. Hydrodynamics appears only on length scales larger than the lattice constant $a$ of the collision-cell lattice. ${ }^{60}$ Consequently, at short times, $t \lesssim 5$ (Fig. 4), the simulation results deviate from the solution of the continuum Navier-Stokes equations, independent of polymer length, as illustrated in Refs. 48, 60, and 82 for various systems. Therefore, agreement between theory and simulations can

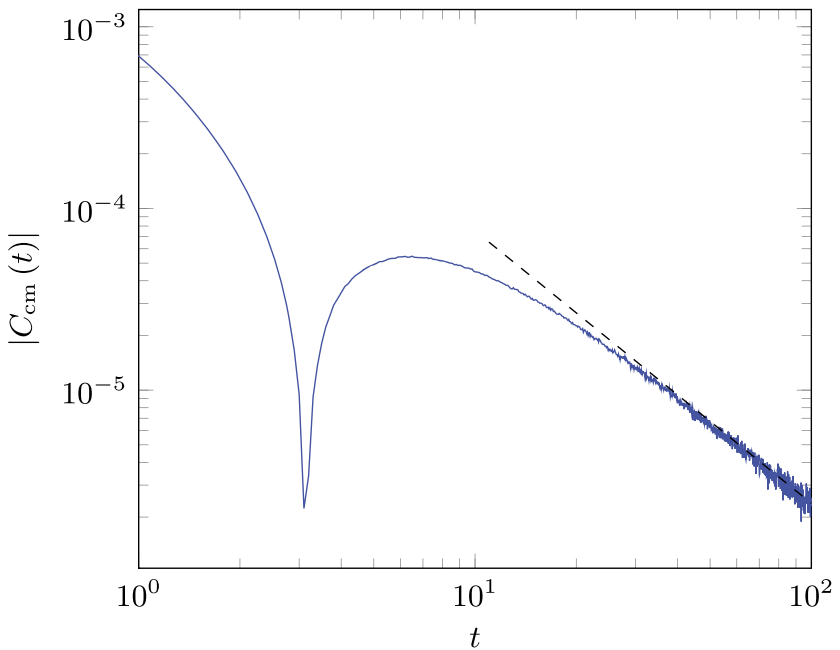

FIG. 4. Time dependence of the velocity autocorrelation function for polymers with $N=100$ monomers and $I=1(K=3)$. The size of the simulation box is $L_{S}=100$. The dashed line indicates the power law $t^{-3 / 2}$.

only be expected at longer times. This does not affect the dynamics of embedded colloids or polymers because it is determined by the hydrodynamic long-time tail.

The correlation function for polymers of length $N=100$ and bond length $l=1$ is presented in Fig. 4. At $t=0, C_{\mathrm{cm}}(0)$ $=3 k_{B} T / m N$, according to the equipartition theorem. (Note that this value includes transverse and longitudinal contributions.) For short times, i.e., for $t \lesssim 1, C_{\mathrm{cm}}(t)$ reflects the discrete-time MPC procedure, with the first collision at $t=h=0.1$. For $t \gtrsim 3$, the correlation function becomes negative due to viscoelasticity, as discussed in Sec. V A 2 [Eq. (59)]. At longer times, the correlation function decays in a power-law manner as $\left|C_{\mathrm{cm}}(t)\right| \sim t^{-3 / 2}$, in agreement with the theoretical expectation for the regime $\tau_{l} \approx 1 \ll t \ll \tau_{R} \approx 6 \times 10^{3}$ (cf. Sec. V A). In the simulations, we did not reach the asymptotic value for $t \rightarrow$ $\infty$ [Eq. (50)], where the correlation function is positive again. The dependence $t^{-3 / 2}$ of the correlation function emphasizes and reflects the relevance of hydrodynamic interactions in a viscoelastic fluid of phantom polymers.

\section{CONCLUSIONS}

We have presented a polymer-based model for a viscoelastic fluid and its implementation in a multiparticle collision dynamics algorithm. The fluid properties have been characterized by the transverse velocity autocorrelation function. The comparison between analytical predictions, based on the Navier-Stokes equation, and simulation results shows good agreement and, thus, confirms the suitability of the applied implementation to describe viscoelastic fluids.

Polymer elasticity strongly affects the velocity correlation function, $\tilde{C}^{T}(\boldsymbol{k}, t)$, and leads to damped oscillations over a certain range of wave vectors. However, for long times and large length scales $(|\boldsymbol{k}| \rightarrow 0)$, we expect and predict an exponential decay of $\tilde{C}^{T}(\boldsymbol{k}, t)$ 
$\sim e^{-v_{f} k^{2} t}$ as a function of time, with the kinematic viscosity of the polymeric fluid. This implies a long-time tail for the polymer centerof-mass velocity correlation function $C_{\mathrm{cm}}(t) \sim t^{-3 / 2}$. On these scales, the polymer melt behaves as a fluid in terms of the correlation functions and, hence, exhibits hydrodynamics with the effective viscosity $\eta_{f}$. More interestingly, for very long polymers, an additional powerlaw time regime can be identified. In the range $\tau_{l}<t<\tau_{R}$ between the relaxation time on the scale of a bond and the Rouse time of the whole polymer, with a nearly continuous mode spectrum, $\tilde{C}^{T}(\boldsymbol{k}, t)$ is negative and exhibits the power-law dependence $t^{-3 / 2}$ rather than an exponential decay with time. Fourier transformation of the correlation function weighted by the static structure factor maintains the time dependence such that the polymer center-of-mass correlation function in real space shows the same time dependence. This rather distinct behavior is a consequence of the wide spectrum of modes and, thus, is a polymer-specific property. It reflects a strong influence of the polymer internal dynamics on the overall hydrodynamic behavior of the fluid. ${ }^{4}$

Here, we have only considered phantom polymers. Extension to polymers with excluded-volume interactions would be interesting, specifically regarding the impact of excluded-volume interactions on the correlation functions. Yet, the results of Ref. 42 show that even then the correlation function of a nonentangled polymer melt exhibits a long-time tail with the decay $t^{-3 / 2}$ in three dimensions.

Our results in the presence of a hydrodynamic long-time tail are consistent with theoretical predictions for phantom polymers. ${ }^{40}$ However, as pointed out in Ref. 42, the studies of self-avoiding polymers seem to contradict the widely accepted view that hydrodynamic interactions are screened in polymer melts. ${ }^{10}$ To be precise, the statement is typically used in the context of polymer solutions, where polymers are dissolved in a fluid, and momentum conservation is assumed to be violated for the fluid due to the immobile polymer matrix. Studies of the dynamical interplay of polymers and fluid would be desirable for a better understanding of screening. The presented simulation approach of polymers and fluid is very well suited for such an endeavor.

\section{ACKNOWLEDGMENTS}

D.T. thanks the Institute of Complex Systems for its hospitality during an extended visit. G.K. and D.T. acknowledge financial support from the Austrian Science Fund (FWF) within the SFB ViCoM (Grant No. F41). D.T. acknowledges financial support from the FWF under Project No. I3846-N36 as well as computing time granted by the Vienna Scientific Cluster. R.G.W. and G.G. gratefully acknowledge the computing time granted through JARA-HPC on the supercomputer JURECA at Forschungszentrum Jülich.

\section{APPENDIX A: GENERAL INVERSE LAPLACE TRANSFORM OF $\hat{C}^{T}(k, s)$}

To calculate the inverse Laplace transform $\tilde{C}^{T}(\boldsymbol{k}, t)$ of $\hat{C}^{T}(\boldsymbol{k}, \boldsymbol{s})$ of Eq. (29), the denominator of the right-hand side,

$$
D(s)=\varrho s+\boldsymbol{k}^{2}\left(\eta+\varphi k_{B} T \sum_{p=1}^{N-1}\left(s+2 / \tau_{n}\right)^{-1}\right),
$$

is multiplied by $W(s)=\prod_{p=1}^{N-1}\left(s+2 / \tau_{n}\right)$, which yields

$$
\begin{aligned}
P(s) & =D(s) W(s) \\
& =\left(\varrho s+\eta \boldsymbol{k}^{2}\right) W(s)+\boldsymbol{k}^{2} \varphi k_{B} T \sum_{n=1}^{N-1} \prod_{\substack{p=1 \\
p \neq n}}^{N-1}\left(s+2 / \tau_{n}\right),
\end{aligned}
$$

a polynomial in $s$ of degree $(N-1)$. Let $P_{n}, n=1, \ldots, M$, be the $M$ distinct roots of $Q^{-1} P(s)$, each of multiplicity $M_{n}$ so that $\sum_{n=1}^{M} M_{n}=N$; hence, $P(s)=\varrho \prod_{m=1}^{M}\left(s-P_{m}\right)^{M_{m}}$. For $W(s)$, which is also a polynomial in $s$, let $W_{n}$ be the coefficient of $s^{n}$ so that $W(s)=\sum_{m=0}^{N-1} W_{n} s^{n}$. Then, (29) can be written as

$$
\begin{aligned}
\hat{C}^{T}(\boldsymbol{k}, s) & =\varrho \tilde{C}^{T}(\boldsymbol{k}, 0) \frac{W(s)}{P(s)} \\
& =\tilde{C}^{T}(\boldsymbol{k}, 0) \sum_{n=0}^{N-1} \frac{W_{n} s^{n}}{\prod_{m=1}^{M}\left(s-P_{m}\right)^{M_{m}}} .
\end{aligned}
$$

Inverse Laplace transformation of the terms $s^{n} \prod_{m=1}^{M}\left(s-P_{m}\right)^{-M_{m}}$ yields ${ }^{83,84}$

$$
\frac{\tilde{C}^{T}(\boldsymbol{k}, t)}{\tilde{C}^{T}(\boldsymbol{k}, 0)}=\sum_{n=0}^{N-1} W_{n} \sum_{m=1}^{M} e^{P_{m} t} \sum_{l=1}^{M_{m}} \frac{A_{n m l}\left(P_{m}\right) t^{M_{m}-l}}{\left(M_{m}-l\right) !(l-1) !},
$$

with

$$
A_{n m l}(x)=\frac{d^{l-1}}{d x^{l-1}}\left(x^{n} \prod_{\substack{j=1 \\ j \neq m}}^{M}\left(x-P_{j}\right)^{-M_{j}}\right) .
$$

Note that the degree of the polynomial of the numerator is higher than the one of the denominator. If $P(s)$ only has simple roots, i.e., $M_{n}=1$ for all $n$, Eq. (A4) simplifies to

$$
\frac{\tilde{C}^{T}(\boldsymbol{k}, t)}{\tilde{\boldsymbol{C}}^{T}(\boldsymbol{k}, 0)}=\sum_{n=0}^{N-1} W_{n} \sum_{m=1}^{N} P_{m}^{n} e^{P_{m} t} \prod_{\substack{j=1 \\ j \neq m}}^{N}\left(P_{m}-P_{j}\right)^{-1} .
$$

\section{APPENDIX B: INVERSE LAPLACE TRANSFORMATION FOR CONTINUOUS POLYMER}

The correlation function (46) is of the form

$$
\hat{f}(s)=\frac{1}{s+b / \sqrt{s}}=\frac{\sqrt{s}}{s^{3 / 2}+b} .
$$

The inverse Laplace-transform $f(t)=\mathcal{L}^{-1}[\hat{f}(s) ; t]$ can be obtained from that of $\hat{H}(\sqrt{s})$, defined as

$$
\hat{H}(s)=\frac{s}{s^{3}+b},
$$

according to ${ }^{78}$

$$
\begin{aligned}
f(t) & =\mathcal{L}^{-1}[\hat{f}(s) ; t]=\mathcal{L}^{-1}[\hat{H}(\sqrt{s}) ; t] \\
& =\frac{1}{2 \sqrt{\pi t^{3}}} \int_{0}^{\infty} \tau \exp -\frac{\tau^{2}}{4 t} H(\tau) d \tau .
\end{aligned}
$$


Partial fraction decomposition of $s^{3}+b$ in $\hat{H}(\sqrt{s})$ and straightforward inverse Laplace transformation yields

$$
H(t)=\frac{1}{3 \sqrt[3]{b}}\left(e^{\kappa t} \cos (\omega t)+\sqrt{3} e^{\kappa t} \sin (\omega t)-e^{-2 \kappa t}\right),
$$

with the abbreviations $\kappa=\sqrt[3]{b} / 2$ and $\omega=\sqrt{3} \sqrt[3]{b} / 2$. Evaluation of the integral (B3) gives, with (B4),

$$
\begin{aligned}
f(x)= & \frac{1}{3}\left\{\operatorname{erfcx}\left(-\frac{1}{2}[1+i \sqrt{3}] \sqrt{x}\right)\right. \\
& \left.+\operatorname{erfcx}\left(-\frac{1}{2}[1-i \sqrt{3}] \sqrt{x}\right)+\operatorname{erfcx}(\sqrt{x})\right\},
\end{aligned}
$$

with $x=b^{2 / 3} t$. Here, $\operatorname{erfcx}(y)$ is the scaled complementary error function,

$$
\operatorname{erfcx}(y)=e^{y^{2}}\left(1-\frac{2}{\sqrt{\pi}} \int_{0}^{y} e^{-u^{2}} d u\right)
$$

We like to mention that Laplace transformation of Eq. (46) including $\eta$ can be performed in a similar manner.

\section{APPENDIX C: CENTER-OF-MASS VELOCITY AUTOCORRELATION FUNCTION-CONNECTION TO DYNAMIC STRUCTURE FACTOR}

The polymer center-of-mass correlation function is given by [cf. Eq. (54)]

$$
C_{\mathrm{cm}}(t)=\frac{1}{N^{2}} \sum_{i=1}^{N} \sum_{j=1}^{N}\left\langle\boldsymbol{v}\left(\boldsymbol{r}_{i}, t\right) \cdot \boldsymbol{v}\left(\boldsymbol{r}_{j}, 0\right)\right\rangle .
$$

The Fourier representation

$$
\boldsymbol{v}\left(\boldsymbol{r}_{i}, t\right)=\frac{1}{(2 \pi)^{2}} \int \tilde{\boldsymbol{v}}(\boldsymbol{k}, t) e^{i \boldsymbol{k} \cdot \boldsymbol{r}_{i}(t)} d^{3} k
$$

yields

$$
C_{\mathrm{cm}}(t)=\frac{1}{N^{2}(2 \pi)^{2}} \sum_{i=1}^{N} \sum_{j=1}^{N}\left\langle\tilde{\boldsymbol{v}}(\boldsymbol{k}, t) \cdot \tilde{\boldsymbol{v}}\left(\boldsymbol{k}^{\prime}, 0\right)\right\rangle e^{i \boldsymbol{k} \cdot \boldsymbol{r}_{i}(t)} e^{i \boldsymbol{k}^{\prime} \cdot \boldsymbol{r}_{j}(0)} d^{3} k d^{3} k^{\prime} .
$$

With the definition of the fluid correlation function $\langle\tilde{\boldsymbol{v}}(\boldsymbol{k}, t)$ . $\left.\tilde{\boldsymbol{v}}\left(\boldsymbol{k}^{\prime}, 0\right)\right\rangle \sim \delta\left(\boldsymbol{k}+\boldsymbol{k}^{\prime}\right) \tilde{C}(\boldsymbol{k}, t)(24)$, and the dynamic structure factor (56),

$$
S(\boldsymbol{k}, t)=\frac{1}{N} \sum_{i=1}^{N} \sum_{j=1}^{N}\left\langle e^{i \boldsymbol{k} \cdot\left(\boldsymbol{r}_{i}(t)-\boldsymbol{r}_{j}(0)\right)}\right\rangle,
$$

Eq. (C1) becomes

$$
C_{\mathrm{cm}}^{T}(t)=\frac{1}{(2 \pi)^{3} N} \int S(\boldsymbol{k}, t) \tilde{C}^{T}(\boldsymbol{k}, t) d^{3} k
$$

which is Eq. (55).

\section{REFERENCES}

${ }^{1}$ Soft Matter: From Synthetic to Biological Materials, Lecture Notes of the 39th IFF Spring School, edited by J. K. G. Dhont, G. Gompper, G. Nägele, D. Richter, and R. G. Winkler (Forschungzentrum Jülich, Jülich, 2008)

${ }^{2}$ A. M. Menzel, “Tuned, driven, and active soft matter," Phys. Rep. 554, 1 (2015).

${ }^{3}$ S. R. Nagel, "Experimental soft-matter science," Rev. Mod. Phys. 89, 025002 (2017).

${ }^{4}$ R. J. Ellis, "Macromolecular crowding: An important but neglected aspect of the intracellular environment," Curr. Opin. Struct. Biol. 11, 114 (2001).

${ }^{5}$ S. Bucciarelli, J. S. Myung, B. Farago, S. Das, G. A. Vliegenthart, O. Holderer, R. G. Winkler, P. Schurtenberger, G. Gompper, and A. Stradner, "Dramatic influence of patchy attractions on short-time protein diffusion under crowded conditions," Sci. Adv. 2, e1601432 (2016).

${ }^{6}$ R. B. Bird, R. C. Armstrong, and O. Hassager, Dynamics of Polymer Liquids (John Wiley \& Sons, New York, 1987), Vol. 1.

${ }^{7}$ J. K. G. Dhont, An Introduction to Dynamics of Colloids (Elsevier, Amsterdam, 1996).

${ }^{8}$ J. D. Ferry, Viscoelastic Properties of Polymers (Wiley, New York, 1980).

${ }^{9}$ R. B. Bird, C. F. Curtiss, R. C. Armstrong, and O. Hassager, Dynamics of Polymer Liquids (John Wiley \& Sons, New York, 1987), Vol. 2.

${ }^{10} \mathrm{M}$. Doi and S. F. Edwards, The Theory of Polymer Dynamics (Clarendon Press, Oxford, 1986).

${ }^{11}$ R. G. Larson, The Structure and Rheology of Complex Fluids (Oxford University, New York, 1999).

${ }^{12}$ R. G. Winkler, D. A. Fedosov, and G. Gompper, "Dynamical and rheological properties of soft colloid suspensions," Curr. Opin. Colloid Interface Sci. 19, 594 (2014).

${ }^{13}$ Y.-G. Tao, I. O. Götze, and G. Gompper, "Multiparticle collision dynamics modeling of viscoelastic fluids," J. Chem. Phys. 128, 144902 (2008).

${ }^{14}$ B. Kowalik and R. G. Winkler, "Multiparticle collision dynamics simulations of viscoelastic fluids: Shear-thinning Gaussian dumbbells,” J. Chem. Phys. 138, 104903 (2013).

${ }^{15}$ G. R. McNamara and G. Zanetti, "Use of the Boltzmann equation to simulate lattice-gas automata," Phys. Rev. Lett. 61, 2332 (1988).

${ }^{16} \mathrm{X}$. Shan and H. Chen, "Lattice Boltzmann model for simulating flows with multiple phases and components," Phys. Rev. E 47, 1815 (1993).

${ }^{17}$ S. Succi, The Lattice Boltzmann Equation: For Fluid Dynamics and Beyond (Oxford University Press, 2001).

${ }^{18}$ B. Dünweg and A. C. Ladd, "Lattice Boltzmann simulations of soft matter systems," Adv. Polym. Sci. 221, 89 (2009).

${ }^{19}$ P. J. Hoogerbrugge and J. M. V. A. Koelman, "Simulating microscopic hydrodynamics phenomena with dissipative particle dynamics," Europhys. Lett. 19, 155 (1992).

${ }^{20} \mathrm{P}$. Español and P. B. Warren, "Statistical mechanics of dissipative particle dynamics," Europhys. Lett. 30, 191 (1995)

${ }^{21}$ A. Malevanets and R. Kapral, "Mesoscopic model for solvent dynamics," J. Chem. Phys. 110, 8605 (1999).

${ }^{22}$ R. Kapral, "Multiparticle collision dynamics: Simulations of complex systems on mesoscale," Adv. Chem. Phys. 140, 89 (2008).

${ }^{23}$ G. Gompper, T. Ihle, D. M. Kroll, and R. G. Winkler, "Multi-particle collision dynamics: A particle-based mesoscale simulation approach to the hydrodynamics of complex fluids," Adv. Polym. Sci. 221, 1 (2009).

${ }^{24}$ I. Ispolatov and M. Grant, "Lattice Boltzmann method for viscoelastic fluids," Phys. Rev. E 65, 056704 (2002).

${ }^{25} \mathrm{P}$. Dellar, "Lattice Boltzmann formulation for linear viscoelastic fluids using an abstract second stress," SIAM J. Sci. Comput. 36, A2507 (2014).

${ }^{26}$ D. A. Fedosov, W. Pan, B. Caswell, G. Gompper, and G. E. Karniadakis, "Predicting human blood viscosity in silico," Proc. Natl. Acad. Sci. U. S. A. 108, 11772 (2011).

${ }^{27}$ D. A. Fedosov, S. P. Singh, A. Chatterji, R. G. Winkler, and G. Gompper, "Semidilute solutions of ultra-soft colloids under shear flow," Soft Matter 8, 4109 (2012). 
${ }^{28}$ C.-C. Huang, R. G. Winkler, G. Sutmann, and G. Gompper, "Semidilute polymer solutions at equilibrium and under shear flow," Macromolecules 43, 10107 (2010).

${ }^{29}$ A. Nikoubashman and C. N. Likos, "Branched polymers under shear," Macromolecules 43, 1610 (2010).

${ }^{30}$ S. P. Singh, A. Chatterji, G. Gompper, and R. G. Winkler, "Dynamical and rheological properties of ultrasoft colloids under shear flow," Macromolecules 46, 8026 (2013).

${ }^{31}$ R. G. Winkler, S. P. Singh, C.-C. Huang, D. A. Fedosov, K. Mussawisade, A. Chatterji, M. Ripoll, and G. Gompper, "Mesoscale hydrodynamics simulations of particle suspensions under shear flow: From hard to ultrasoft colloids," Eur. Phys. J.: Spec. Top. 222, 2773 (2013).

${ }^{32} \mathrm{D}$. Toneian, "Magnetically functionalized star polymers and polymer melts in MPCD simulations," Ph.D. thesis, TU Wien, 2019.

${ }^{33}$ D. Toneian, C. N. Likos, and G. Kahl, "Controlled self-aggregation of polymer-based nanoparticles employing shear flow and magnetic fields," J. Phys.: Condens. Matter 31, 24LT02 (2019); e-print arXiv:1904.01535 [cond-mat.soft].

${ }^{34} \mathrm{H}$. Noguchi and G. Gompper, "Fluid vesicles with viscous membranes in shear flow,” Phys. Rev. Lett. 93, 258102 (2004).

${ }^{35}$ E. Somfai, A. Morozov, and W. van Saarloos, "Modeling viscoelastic flow with discrete methods," Physica A 362, 93 (2006).

${ }^{36}$ D. A. Fedosov, G. E. Karniadakis, and B. Caswell, "Steady shear rheometry of dissipative particle dynamics models of polymer fluids in reverse Poiseuille flow," J. Chem. Phys. 132, 144103 (2010).

${ }^{37} \mathrm{~S}$. Ji, R. Jiang, R. G. Winkler, and G. Gompper, "Mesoscale hydrodynamic modeling of a colloid in shear-thinning viscoelastic fluids under shear flow," J. Chem. Phys. 135, 134116 (2011).

${ }^{38}$ D. Toneian, "Multi-particle collision dynamics simulation of viscoelastic fluids," M.S. thesis, TU Wien, 2015.

${ }^{39}$ P.-G. de Gennes, Scaling Concepts in Polymer Physics (Cornell University, Ithaca, 1979).

${ }^{40}$ K. F. Freed and A. Perico, "Considerations on the multiple scattering representation of the concentration dependence of the viscoelastic properties of polymer systems," Macromolecules 14, 1290 (1981).

${ }^{41} \mathrm{~J}$. Farago, H. Meyer, and A. N. Semenov, "Anomalous diffusion of a polymer chain in an unentangled melt," Phys. Rev. Lett. 107, 178301 (2011).

${ }^{42}$ J. Farago, H. Meyer, J. Baschnagel, and A. N. Semenov, "Mode-coupling approach to polymer diffusion in an unentangled melt. II. The effect of viscoelastic hydrodynamic interactions," Phys. Rev. E 85, 051807 (2012).

${ }^{43} \mathrm{~K}$. Mussawisade, M. Ripoll, R. G. Winkler, and G. Gompper, "Dynamics of polymers in a particle based mesoscopic solvent," J. Chem. Phys. 123, 144905 (2005).

${ }^{44}$ J. F. Ryder and J. M. Yeomans, "Shear thinning in dilute polymer solutions," J. Chem. Phys. 125, 194906 (2006).

${ }^{45}$ S. Frank and R. G. Winkler, "Polyelectrolyte electrophoresis: Field effects and hydrodynamic interactions," Europhys. Lett. 83, 38004 (2008).

${ }^{46}$ R. Chelakkot, R. G. Winkler, and G. Gompper, "Flow-induced helical coiling of semiflexible polymers in structured microchannels," Phys. Rev. Lett. 109, 178101 (2012).

${ }^{47}$ A. Nikoubashman and C. N. Likos, "Flow-induced polymer translocation through narrow and patterned channels," J. Chem. Phys. 133, 074901 (2010).

${ }^{48}$ C. C. Huang, G. Gompper, and R. G. Winkler, "Effect of hydrodynamic correlations on the dynamics of polymers in dilute solution," J. Chem. Phys. 138, 144902 (2013).

${ }^{49}$ M. Ripoll, R. G. Winkler, and G. Gompper, "Star polymers in shear flow," Phys. Rev. Lett. 96, 188302 (2006).

${ }^{50}$ S. P. Singh, C.-C. Huang, E. Westphal, G. Gompper, and R. G. Winkler, "Hydrodynamic correlations and diffusion coefficient of star polymers in solution," J. Chem. Phys. 141, 084901 (2014).

${ }^{51}$ A. Ghavami and R. G. Winkler, "Solvent induced inversion of core-shell microgels,” ACS Macro Lett. 6, 721 (2017).
${ }^{52}$ E. Westphal, S. P. Singh, C.-C. Huang, G. Gompper, and R. G. Winkler, "Multiparticle collision dynamics: GPU accelerated particle-based mesoscale hydrodynamic simulations," Comput. Phys. Commun. 185, 495 (2014).

${ }^{53}$ M. P. Howard, A. Z. Panagiotopoulos, and A. Nikoubashman, "Efficient mesoscale hydrodynamics: Multiparticle collision dynamics with massively parallel GPU acceleration," Comput. Phys. Commun. 230, 10 (2018).

${ }^{54} \mathrm{~B}$. U. Felderhof, "Backtracking of a sphere slowing down in a viscous compressible fluid," J. Chem. Phys. 123, 044902 (2005).

${ }^{55} \mathrm{~B}$. J. Alder and T. E. Wainwright, "Decay of the velocity autocorrelation function," Phys. Rev. A 1, 18 (1970).

${ }^{56} \mathrm{R}$. Zwanzig and M. Bixon, "Hydrodynamic theory of the velocity correlation function," Phys. Rev. A 2, 2005 (1970).

${ }^{57}$ M. H. Ernst, E. H. Hauge, and J. M. J. van Leeuwen, "Asymptotic time behavior of correlation functions. I. Kinetic terms,” Phys. Rev. A 4, 2055 (1971).

${ }^{58}$ E. Hauge and A. Martin-Löf, "Fluctuating hydrodynamics and Brownian motion," J. Stat. Phys. 7, 259 (1973).

${ }^{59}$ E. J. Hinch, "Application of the Langevin equation to fluid suspensions," J. Fluid Mech. 72, 499 (1975).

${ }^{60}$ C.-C. Huang, G. Gompper, and R. G. Winkler, "Hydrodynamic correlations in multiparticle collision dynamics fluids," Phys. Rev. E 86, 056711 (2012).

${ }^{61}$ R. G. Winkler and P. Reineker, "Finite size distribution and partition functions of Gaussian chains: Maximum entropy approach," Macromolecules 25, 6891 (1992).

${ }^{62}$ L. Harnau, R. G. Winkler, and P. Reineker, "Influence of stiffness on the dynamics of macromolecules in a melt," J. Chem. Phys. 106, 2469 (1997).

${ }^{63} \mathrm{P}$. H. Verdier, "Monte Carlo studies of lattice-model polymer chains. I. Correlation functions in the statistical-bead model," J. Chem. Phys. 45, 2118 (1966).

${ }^{64}$ A. Kopf, B. Dünweg, and W. Paul, "Dynamics of polymer "isotope" mixtures: Molecular dynamics simulation and Rouse model analysis," J. Chem. Phys. 107, 6945 (1997).

${ }^{65}$ R. G. Winkler, "Analytical calculation of the relaxation dynamics of partially stretched flexible chain molecules: Necessity of a wormlike chain description," Phys. Rev. Lett. 82, 1843 (1999).

${ }^{66} \mathrm{R}$. G. Winkler, "Conformational and rheological properties of semiflexible polymers in shear flow," J. Chem. Phys. 133, 164905 (2010).

${ }^{67}$ L. D. Landau and E. M. Lifshitz, Fluid Mechanics (Pergamon Press, London, 1959).

${ }^{68}$ D. Frenkel and B. Smit, Understanding Molecular Simulation, 2nd ed. (Academic Press, 2002).

${ }^{69}$ T. Ihle and D. M. Kroll, "Stochastic rotation dynamics: A Galilean-invariant mesoscopic model for fluid flow,” Phys. Rev. E 63, 020201(R) (2001).

${ }^{70}$ M. Ripoll, K. Mussawisade, R. G. Winkler, and G. Gompper, "Dynamic regimes of fluids simulated by multi-particle-collision dynamics," Phys. Rev. E 72, 016701 (2005).

${ }^{71}$ E. Tüzel, T. Ihle, and D. M. Kroll, "Dynamic correlations in stochastic rotation dynamics," Phys. Rev. E 74, 056702 (2006).

${ }^{72}$ C. Huang, A. Chatterji, G. Sutmann, G. Gompper, and R. G. Winkler, "Celllevel canonical sampling by velocity scaling for multiparticle collision dynamics simulations," J. Comput. Phys. 229, 168-177 (2010).

${ }^{73} \mathrm{D}$. Toneian, OpenMPCD, https://openmpcd.org.

${ }^{74} \mathrm{~A}$. Malevanets and R. Kapral, "Solute molecular dynamics in a mesoscale solvent," J. Chem. Phys. 112, 7260 (2000).

${ }^{75}$ CUDA C programming Guide, Version 7.5, NVIDIA, 2015.

${ }^{76}$ C.-C. Huang, A. Varghese, G. Gompper, and R. G. Winkler, "Thermostat for nonequilibrium multiparticle-collision-dynamics simulations," Phys. Rev. E 91, 013310 (2015).

${ }^{77}$ P. Dyke, in An Introduction to Laplace Transforms and Fourier Series, Springer Undergraduate Mathematics Series, 2nd ed., edited by M. A. J. Chaplain, K. Erdmann, A. MacIntyre, E. Süli, M. R. Tehranchi, and J. F. Toland (Springer, 2014).

${ }^{78}$ F. Oberhettinger and L. Badii, Tables of Laplace Transforms (Springer Science \& Business Media, 2012). 
${ }^{79}$ R. G. Winkler, S. Keller, and J. O. Rädler, "Intramolecular dynamics of linear macromolecules by fluorescence correlation spectroscopy," Phys. Rev. E 73, 041919 (2006)

${ }^{80}$ L. Harnau, R. G. Winkler, and P. Reineker, "Dynamic structure factor of semiflexible macromolecules in dilute solution," J. Chem. Phys. 104, 6355 (1996).

${ }^{81}$ R. G. Winkler, L. Harnau, and P. Reineker, "Distribution functions and dynamical properties of stiff macromolecules," Macromol. Theory Simul. 6, 1007 (1997).
${ }^{82}$ S. Poblete, A. Wysocki, G. Gompper, and R. G. Winkler, "Hydrodynamics of discrete-particle models of spherical colloids: A multiparticle collision dynamics simulation study," Phys. Rev. E 90, 033314 (2014).

${ }^{83}$ A. Erdélyi, W. Magnus, F. Oberhettinger, and F. G. Tricomi, Tables of Integral Transforms (McGraw-Hill, 1954), Vol. 1.

${ }^{84}$ A. P. Prudnikov, Y. A. Brychkov, and O. I. Marichev, Integrals and Series (Gordon and Breach Science Publishers, 1992), Vol. 5. 